\title{
Clinical, histological, and deep learning-based assessments and treatment of oral verruciform xanthoma: a case report
}

\author{
Jae-Hong Lee ${ }^{1 \star}$ and Seong-Nyum Jeong ${ }^{2}$
}

${ }^{1}$ Associate Professor, Department of Periodontology, Daejeon Dental Hospital, Institute of Wonkwang Dental Research, Wonkwang University College of Dentistry, Daejeon, Republic of Korea

${ }^{2}$ Professor, Department of Periodontology, Daejeon Dental Hospital, Institute of Wonkwang Dental Research, Wonkwang University College of Dentistry, Daejeon, Republic of Korea

\begin{abstract}
Verruciform xanthoma is a benign growth found on the oral mucosa. Clinically, verruciform xanthoma is an asymptomatic lesion, and depending on the amount of keratin present on the surface of the oral mucosa, it can appear as a reddish-gray papillary, flat lesion or a slightly raised, rough lesion. The verrucous structure and exophytic properties of these lesions are similar to those of malignant tumors; therefore, histopathological assessment is required for an accurate and definitive diagnosis. The purpose of this case report is to present the clinical, histological, and deep learning-based assessments and treatment of verruciform xanthoma of the maxillary palatal gingiva.
\end{abstract}

Key Words: Biopsy; Deep learning; Hard palate; Immunohistochemistry; Xanthomatosis

(c) This is an open-access article distributed under the terms of the Creative Commons Attribution Non-Commercial License (http://creativecommons.org/licenses/by-nc/4.0) which permits unrestricted noncommercial use, distribution, and reproduction in any medium, provided the original work is properly cited.

\section{Introduction}

A xanthoma is defined as a yellowish, slightly raised or flat, and fairly well-defined lesion that occurs below the surface of the epithelium [1,2]. Many xanthomas are cutaneous manifestations of generalized or local disease, and most are found to be associated with metabolic disturbances of different lipids. Xanthomas, however, are rare, and the etiology and pathophysiology are still unknown and unclear [1].

Verruciform xanthoma (VX) is a benign growth that can be found on the oral mucosa, often appearing as a pink, yellow, or gray raised plaque or papule with granules, papilla, or verrucous surface morphologies. Due to the rarity of these growths, the etiology and pathogenesis are often unknown [3,4]. VX is often asymptomatic and slowgrowing, and more frequently occurs as a solitary lesion, although multiple lesions have occasionally been reported [5].

VX is seen slightly more in Caucasian males, and is most commonly seen in the 50-60 year age range [6,7]. It is also associated with other oral diseases, including oral pemphigus vulgaris, dysplasia, and lichen planus [6]. Although VX most often appears on the gingiva or alveolar mucosa, several cases have been observed on the floor of the mouth, buccal mucosa, or tongue [4].

Received July 12, 2021; Revised July 31, 2021; Accepted August 8, 2021

*Corresponding author: Jae-Hong Lee, Department of Periodontology, Daejeon Dental Hospital, Wonkwang University College of Dentistry, 77 Dunsan-ro, Seo-gu, Daejeon 35233, Republic of Korea.

Tel: +82-42-366-1114, Fax: +82-42-366-1115, E-mail: ljaehong@gmail.com

Copyright ( ) 2021, Oral Biology Research Institute 
The differential diagnosis for these cases is important because VX can be clinically similar to a malignant tumor [6]. When treating VX, excision is usually therapeutic, and recurrence is rarely reported [5,7]. The purpose of this case report is to present the clinical, histological, and deep learning-based assessment and treatment of VX on the maxillary palatal gingiva.

\section{Ethics, consent, and permissions}

This study was approved by the Institutional Review Board of the Daejeon Dental Hospital at Wonkwang University (approval no. W2007/005-001). Informed consent was obtained in accordance with the Declaration of Helsinki and the guidelines for Good Clinical Practice, and our study follows the CARE reporting guidelines.

\section{Case Description}

A 58-year-old male patient presented to the Department of Periodontology at the Daejeon Dental Hospital with a wart-like nodule on the left palatal attached gingiva. The patient reported spontaneous discomfort on the gingiva, but no specific pain or symptoms for one or two years. The nodule, however began to grow about two months prior to his visit to the dental hospital, particularly in the time right before his visit. The patient had a medical history of pituitary neoplastic syndrome, but was not receiving care at the time of his visit; he also had a smoking history of half a pack a day.

\section{Clinical and radiographic examination}

Clinically, the patient's oral hygiene was fairly good; however, supra- and subgingival calculi were present, as well as tobacco stains. An intraoral examination revealed a well-defined papule with a verrucous surface and reddish color, measuring $1.5 \mathrm{~cm} \times 1.0 \mathrm{~cm}$ on the left maxillary palatal gingiva in the area of the first and second molars. Clinical signs of acute or chronic periodontitis, such as tenderness to palpation, bleeding with probing, deep probing depth (over $3 \mathrm{~mm}$ ), and suppuration were not seen (Fig. 1A). Additionally, periapical radiological examination revealed no signs of chronic inflammation, such as loss of marginal alveolar bone or increased tooth mobility (Fig. 1B).

\section{Incisional biopsy}

An incisional biopsy was performed with a 6-mm biopsy punch (SFM Medical Devices, Wächtersbach, Germany) under local anesthesia (2\% lidocaine HCL with 1:100,000 epinephrine; Yuhan, Seoul, Korea). The excised tissue was immersed in 10\% buffered formalin and sent to the Department of Oral Pathology at the Wonkwang University College of Dentistry for routine histopathological evaluation (Fig. 2). The patient was provided with the following postoperative medications to be used after the biopsy: antibiotics (amoxicillin $500 \mathrm{mg}$ TID for 3 days), analgesics (ibuprofen $200 \mathrm{mg}$ TID for 3 day), and chlorhexidine mouthwash (BID for 2 weeks).
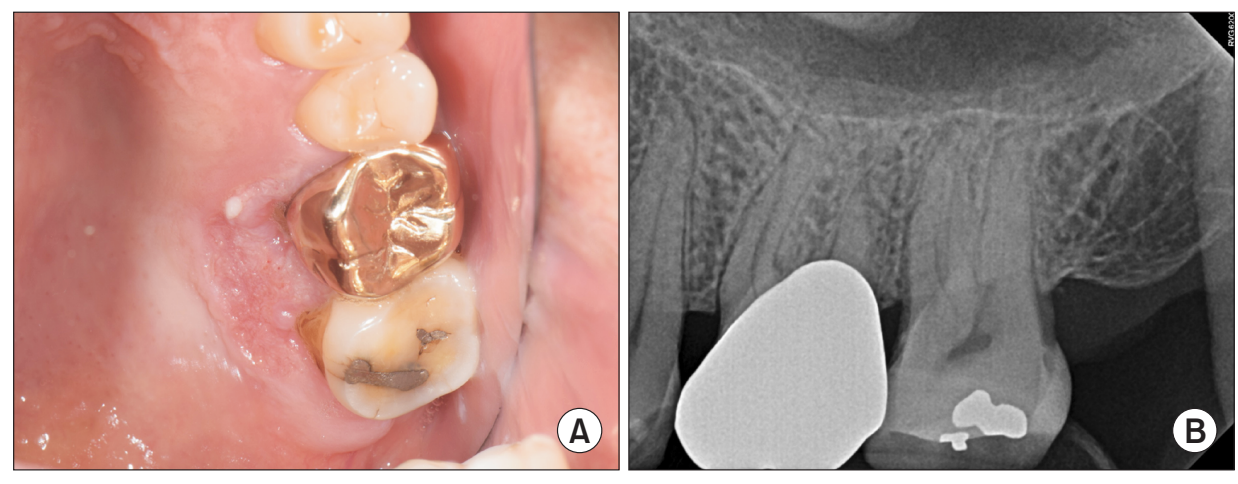

Fig. 1. (A) Clinical photograph on initial examination. Well-defined papillomatous and verrucous surface, measuring $1.5 \mathrm{~cm} \times 1.0 \mathrm{~cm}$ at the left palatal attached gingiva in the area of the first molar. (B) Periapical radiograph on initial examination. No specific or chronic inflammatory signs, including alveolar bone loss or increased tooth mobility. 

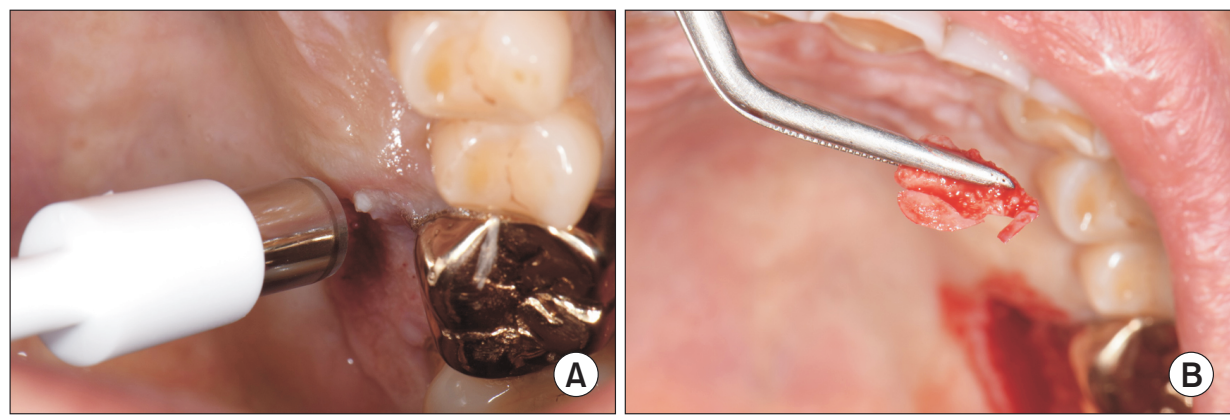

Fig. 2. (A) Incisional biopsy was performed under local anesthesia. (B) The excised specimen was immersed in $10 \%$ buffered formalin for the histopathological examination.

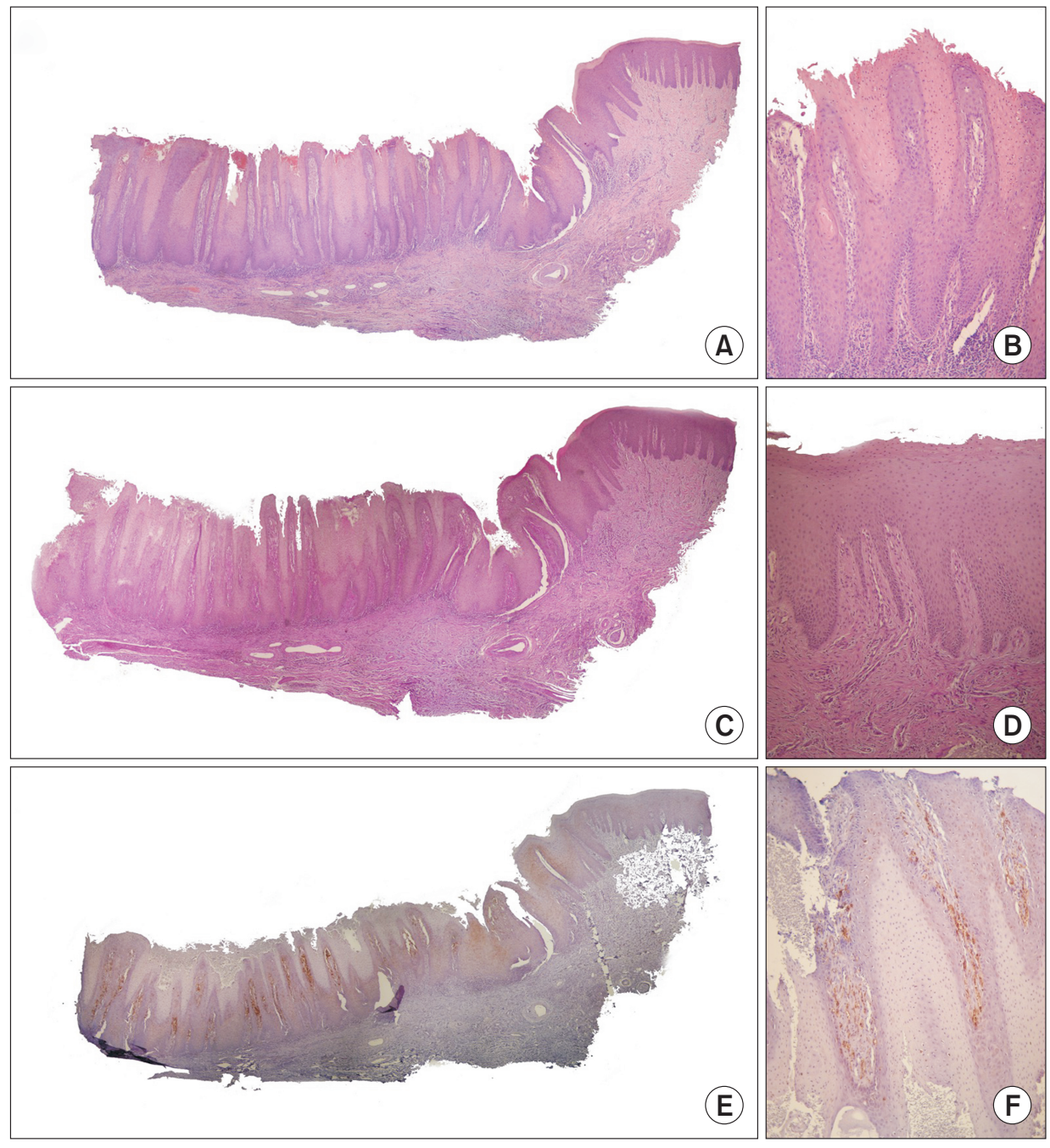

Fig. 3. result of histopathological examination, including hematoxylin and eosin (H\&E) and periodic acid-Schiff (PAS) staining, shows hyperplasia with marked surface parakeratosis, as well as wide elongation of the epidermal rete pegs and acanthosis. Immunohistochemical staining for CD68 shows positive findings in foam cells below the epithelium. The specimen was stained with $\mathrm{H} \& \mathrm{E}(\mathrm{A}: \times 40$ and $\mathrm{B}$ : $\times 100)$, PAS (C: $\times 40$ and $\mathrm{D}: \times 100)$, and anti-CD68 $(\mathrm{E}: \times 40$ and $\mathrm{F}: \times 100)$.

\section{Histopathological findings}

The specimen sent for biopsy consisted of a piece of tissue measuring $0.5 \mathrm{~cm} \times 0.3 \mathrm{~cm}$. The results of histopathological examination, which included hematoxylin and eosin (H\&E) and periodic acid-Schiff (PAS) staining, revealed the presence of foam cells and typical histological features of VX. The verrucous surface of the papule showed hyperplasia with marked surface parakeratosis, as well as wide elongation of the epidermal rete pegs and acanthosis. Additional immunohistochemical staining for CD68 showed positive findings in subepithelial foam cells (Fig. 3). 


\section{Deep learning-based histological assessment}

For the deep learning-based histological assessment, we used positive and negative VX histological images created by web image mining. The dataset consisted of a total of 2,770 patches of $200 \times 200$ pixels (extracted from 590 histological slide images), of which 1,320 were positive and 1,450 were negative for VX. For the assessment, we employed an automated deep convolutional neural network (DCNN) model derived from Auto-Keras (DATA Lab at Texas A\&M University, College Station, TX, USA) and Neuro-T version 1.4.3 (Neurocle Inc., Seoul, Korea) (Fig. 4) [8,9]. The performance metrics of the automated DCNN based on the accuracy, sensitivity, specificity, and area under the curve showed $0.91 \pm 0.07,0.86 \pm 0.05,0.95 \pm 0.08$, and $0.92(95 \%$ confidence interval [CI] 0.88-0.95), respectively.

\section{Excisional treatment and follow-up}

The lesion was completely excised under local anesthesia, and the wound was sutured with 4-0 e-PTFE (Biotex; Purgo biologics, Seongnam, Korea). The patient returned after two weeks for a follow-up and stitch removal. After four months of monitoring, the lesion appeared to be moderately healed, with no recurrence requiring further treatment (Fig. 5).

\section{Discussion}

VX is a benign and nondestructive lesion that features proliferation of non-Langerhans, lipid-rich histiocytes (foam cells) $[3,10]$. Clinically, VX is an asymptomatic lesion, and depending on the amount of keratin on the surface, it can appear as a reddish- gray papillary, flat, or slightly raised,

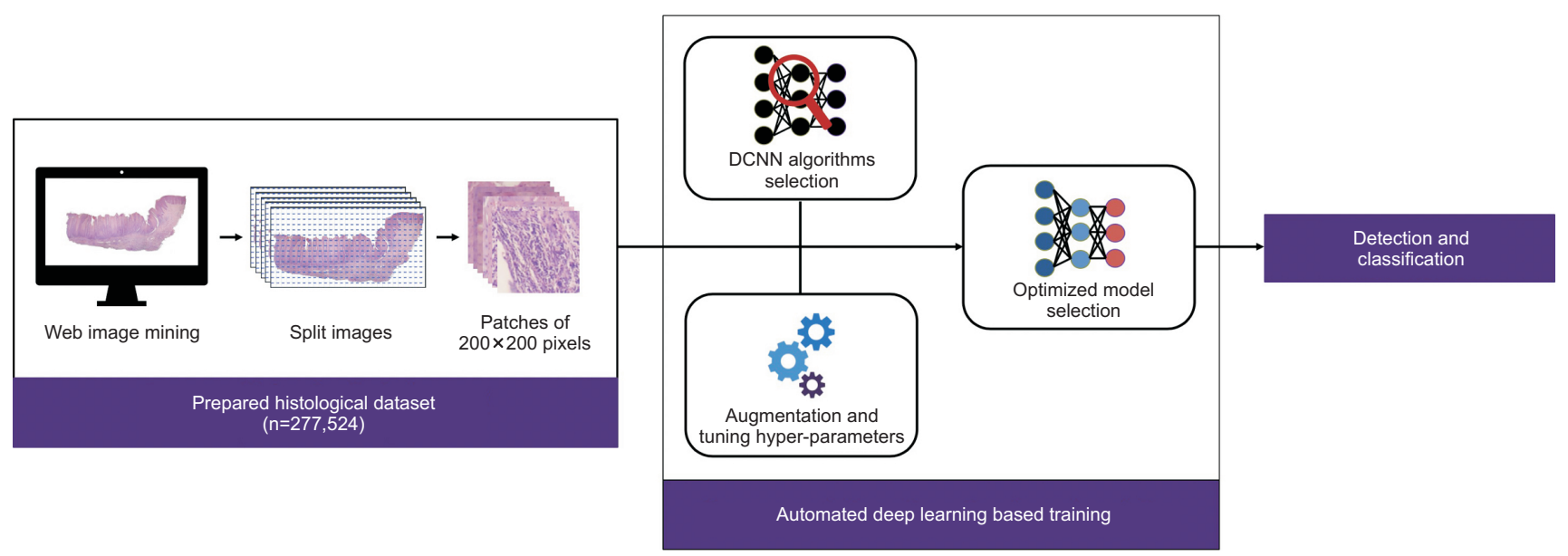

Fig. 4. Overview representing the automated deep learning architecture. The dataset consisted of a total of 2,770 patches of $200 \times 200$ pixels (extracted from 590 histological slide images), consisting of 1,320 positive for and 1,450 negative for verrucous xanthoma.

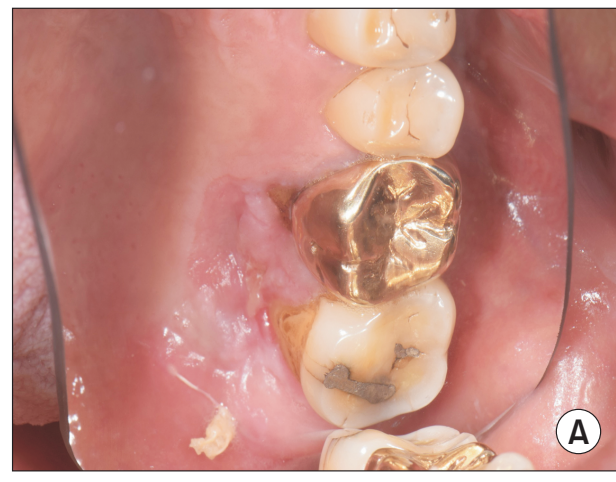

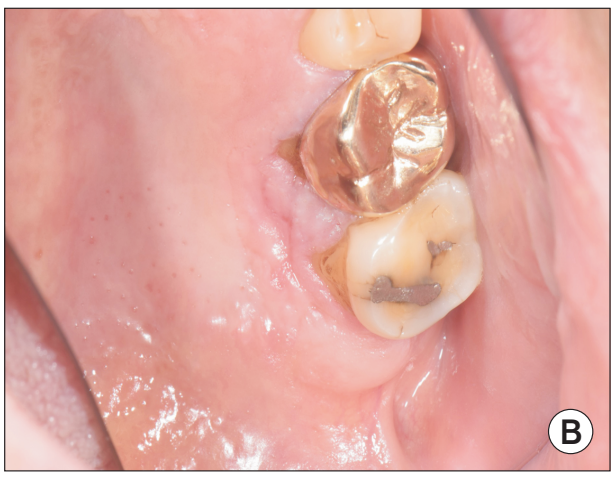

Fig. 5. (A) Clinical photograph at 2 weeks post-excision. (B) Four months post-excision, moderately healed with no recurrence. 
rough lesion $[1,11]$. VX responds well to conservative excision and healing by secondary intention. Recurrence and/ or malignant transformation of VX are exceedingly rare [12]. However, the verrucous structure and exophytic properties of the lesions are similar to malignant tumors, so histopathological assessment is required for an accurate and definitive diagnosis [3].

VX requires a differential diagnosis to include verruca vulgaris, verrucous carcinoma, and squamous cell carcinoma arising within verruca vulgaris, which is associated with squamous proliferation [13]. Verrucous carcinoma is a rare variant of squamous cell carcinoma, which is characterized by microscopically pronounced papillomatosis and marked acanthosis [13]. The absence of significant numbers of xanthoma cells is helpful in distinguishing these lesions from VX [14]. The most prominent histological feature of VX is the presence of large foam cells in the connective tissue papillae, which is seen in all xanthomas, and VX in particular shows hyperkeratosis, uniform epithelial acanthosis, and the development of foam cells within the elongated dermal papillae $[1,11]$. Under low magnification microscopy, a VX specimen shows various features, such as a warty or verrucous appearance, a papillary or cauliflower-like texture, and it can be slightly raised or flat [1].

Immunologically, xanthoma cells from VX contain almost no PAS-positive, diastase-resistant material and are negative for the antibody against S100; however, they are positive for the antibody against CD68 [14]. The S100 antibody may be useful for the differential diagnosis of certain reactive and neoplastic conditions, and is considered as a preferential marker of VX $[4,14]$. Since VX is negative for the antibody against $\mathrm{S100}$, it can be differentially diagnosed with a granular cell tumor in which the S100 protein is expressed [15]. Intense cytoplasmic positivity was observed in VX foam cells using CD68 monoclonal antibodies, which is consistent with monocyte macrophage lineage [4]. In addition, foam cells have been reported to stain positively for cathepsin B, another macrophage marker [16].

Unlike the pre-trained DCNN algorithms that are mainly used in the medical and dental fields (such as VGG16, GoogLeNet Inception, ResNet, SqueezeNet, and MobileNet), for this case report we used an automated DCNN algorithm that finds the optimization of model parameters (includ- ing the number of connected layers, selection of activation function, learning rate, batch size, and drop-out rate) by itself [17-21]. The results showed that our histopathological slides were effectively detected and diagnosed using the automated DCNN architecture. However, the diagnostic accuracy $(0.91,95 \%$ CI $0.87-0.95)$ of VX was less than that found when using conventional histopathological assessment. If we built a well-designed DCNN model with a large number of high-quality datasets, the deep learning-based histological assessment would be expected to be useful enough in clinical practice.

\section{Acknowledgements}

This work was supported by a National Research Foundation of Korea (NRF) grant funded by the Korean government (MSIT) (No. 2019R1A2C1083978).

\section{Conflicts of Interest}

The authors declare that they have no competing interests.

\section{ORCID}

\author{
Jae-Hong Lee \\ https://orcid.org/0000-0002-2375-0141 \\ Seong-Nyum Jeong \\ https://orcid.org/0000-0003-4890-989X
}

\section{References}

1. Nowparast B, Howell FV, Rick GM. Verruciform xanthoma. A clinicopathologic review and report of fifty-four cases. Oral Surg Oral Med Oral Pathol 1981;51:619-625. doi: 10.1016/s0030-4220(81)80012-6.

2. Kim DY, Baik U, Jeon JH. Osteogenesis imperfecta and combined orthodontics and orthognathic surgery: a case report on two siblings. J Korean Assoc Oral Maxillofac Surg 2020;46:70-77. doi: 10.5125/jkaoms.2020.46.1.70.

3. Harris L, Staines K, Pring M. Oral verruciform xanthoma. BMJ Case Rep 2015;2015:bcr2014209216. doi: 10.1136/ bcr-2014-209216.

4. Mostafa KA, Takata T, Ogawa I, Ijuhin N, Nikai H. Verruciform xanthoma of the oral mucosa: a clinicopathological study with immunohistochemical findings relating to pathogenesis. Virchows Arch A Pathol Anat Histopathol 
1993;423:243-248. doi: 10.1007/BF01606886.

5. Philipsen HP, Reichart PA, Takata T, Ogawa I. Verruciform xanthoma--biological profile of 282 oral lesions based on a literature survey with nine new cases from Japan. Oral Oncol 2003;39:325-336. doi: 10.1016/s1368-8375(02)00088$\mathrm{x}$.

6. Dorankula SP, Ramani P, Premkumar P, Anuja, Sherlyn HJ. Verruciform xanthoma of the oral cavity - a case report. J Clin Diagn Res 2013;7:1799-1801. doi: 10.7860/ JCDR/2013/6559.3309.

7. Yu CH, Tsai TC, Wang JT, Liu BY, Wang YP, Sun A, Chiang CP. Oral verruciform xanthoma: a clinicopathologic study of 15 cases. J Formos Med Assoc 2007;106:141-147. doi: 10.1016/S0929-6646(09)60230-8.

8. Zoph B, Le QV. Neural architecture search with reinforcement learning. ArXiv Forthcoming 2017.

9. Truong A, Walters A, Goodsitt J, Hines K, Bruss CB, Farivar R. Towards automated machine learning: evaluation and comparison of AutoML approaches and tools. ArXiv Forthcoming 2019.

10. Parker F. Xanthomas and hyperlipidemias. J Am Acad Dermatol 1985;13:1-30. doi: 10.1016/s0190-9622(85)701399.

11. Snider RL. Verruciform xanthomas and lymphedema. J Am Acad Dermatol 1992;27(6 Pt 1):1021-1023. doi: 10.1016/ s0190-9622(08)80274-5.

12. van der Waal I, Kerstens HC, Hens CJ. Verruciform xanthoma of the oral mucosa. J Oral Maxillofac Surg 1985;43:623626. doi: 10.1016/0278-2391(85)90134-x.

13. Philippou P, Kitsios C, Miliatou M, Poullou C, Konstantinou P. Organ-sparing surgery for a giant verrucous carcinoma of the penile shaft: a case report and review of the literature. Case Rep Urol 2019;2019:1537379. doi: $10.1155 / 2019 / 1537379$.

14. Reich O, Regauer S. Recurrent verruciform xanthoma of the vulva. Int J Gynecol Pathol 2004;23:75-77. doi: 10.1097/01.pgp.0000101143.79462.f7.

15. Regezi JA, Zarbo RJ, Courtney RM, Crissman JD. Immunoreactivity of granular cell lesions of skin, mucosa, and jaw. Cancer 1989;64:1455-1460. doi: 10. 1002/1097-0142(19891001)64:7〈1455::aid-cncr282064 0716>3.0.co;2-\#.

16. Iamaroon A, Vickers RA. Characterization of verruciform xanthoma by in situ hybridization and immunohistochemistry. J Oral Pathol Med 1996;25:395-400. doi: 10.1111/ j.1600-0714.1996.tb00285.x.

17. Gulshan V, Peng L, Coram M, Stumpe MC, Wu D, Narayanaswamy A, Venugopalan S, Widner K, Madams T, Cuadros J, Kim R, Raman R, Nelson PC, Mega JL, Webster DR. Development and validation of a deep learning algorithm for detection of diabetic retinopathy in retinal fundus photographs. JAMA 2016;316:2402-2410. doi: 10.1001/ jama.2016.17216.

18. Lee JH, Kim DH, Jeong SN, Choi SH. Diagnosis and prediction of periodontally compromised teeth using a deep learning-based convolutional neural network algorithm. J Periodontal Implant Sci 2018;48:114-123. doi: 10.5051/ jpis.2018.48.2.114.

19. Lee JH, Kim DH, Jeong SN, Choi SH. Detection and diagnosis of dental caries using a deep learning-based convolutional neural network algorithm. J Dent 2018;77:106-111. doi: 10.1016/j.jdent.2018.07.015.

20. Lee JH, Kim DH, Jeong SN. Diagnosis of cystic lesions using panoramic and cone beam computed tomographic images based on deep learning neural network. Oral Dis 2020;26:152-158. doi: 10.1111/odi.13223.

21. Lee JH, Jeong SN. Efficacy of deep convolutional neural network algorithm for the identification and classification of dental implant systems, using panoramic and periapical radiographs: a pilot study. Medicine (Baltimore) 2020;99:e20787. doi: 10.1097/MD.00000000000020787. 